\title{
In-Situ Spectrum Imaging with Synchronized and Automated Stimulus Control
}

Liam Spillane ${ }^{1}$, Benjamin Miller ${ }^{2}$, Bernhard Schaffer ${ }^{3}$, Paul Thomas ${ }^{1}$ and Ray Twesten ${ }^{3}$

${ }^{1}$ Gatan Inc., Pleasanton, California, United States, ${ }^{2}$ Gatan Inc., California, United States, ${ }^{3}$ Gatan Inc., United States

Spectrum imaging (SI) performed in the scanning transmission electron microscope (STEM) is a well proven technique for advanced materials characterization due to the wide variety of signals provided, combined with the high spatial resolution provided by the STEM. Morphological and crystallographic information may be determined from imaging and diffraction methods such as 4DSTEM, where composition may be determined from spectroscopic techniques such as energy dispersive ( $\mathrm{x}$-ray) spectroscopy (EDS) and electron-energy loss spectroscopy (EELS). EELS has the further capability of probing local electronic structure, enabling local measurement of chemical bonding, changes in oxidation state, phonon and/or electronic band structure characterization.

For in-situ STEM analysis, the capability to capture data at: high speed, high dose efficiency, and high insitu stimulus resolution is critical. A CMOS based transmission electron counting detector incorporated into an optimized post-column energy filter is a well proven platform for performing both STEM EELS and 4DSTEM experiments. Synchronization of an in-situ stimulus to a multiple pass SI experiment has also previously been demonstrated as an effective methodology for maximizing the in-situ stimulus resolution [1].

Here we use metal oxide systems as model materials systems and present in-situ heating results acquired using a CMOS based electron counting EELS spectrometer (GIF Continuum). These results are used to demonstrate recent advances in synchronized in-situ spectrum imaging acquisition. These advances allow automated acquisition of CBED and EDS signals at a given in-situ condition in addition to the EELS signal. Furthermore, datasets are streamed/written to disk instead of held in memory over the course of the in-situ experiment, following the automated steps shown in figure 1. The combination of these two key advances maximizes the information that can be extracted from a single sample even for irreversible transformations since all data can be acquired in a single experiment, in addition to maximizing the stimulus resolution at which the data can be captured.

Example dual-EELSTM, energy filtered diffraction and EDS multipass spectrum image results acquired from silver (II) oxide over a temperature range of $25-600^{\circ} \mathrm{C}$ are shown in in figure 2 . Temperature control was implemented as per the flow diagram shown in figure 1. 


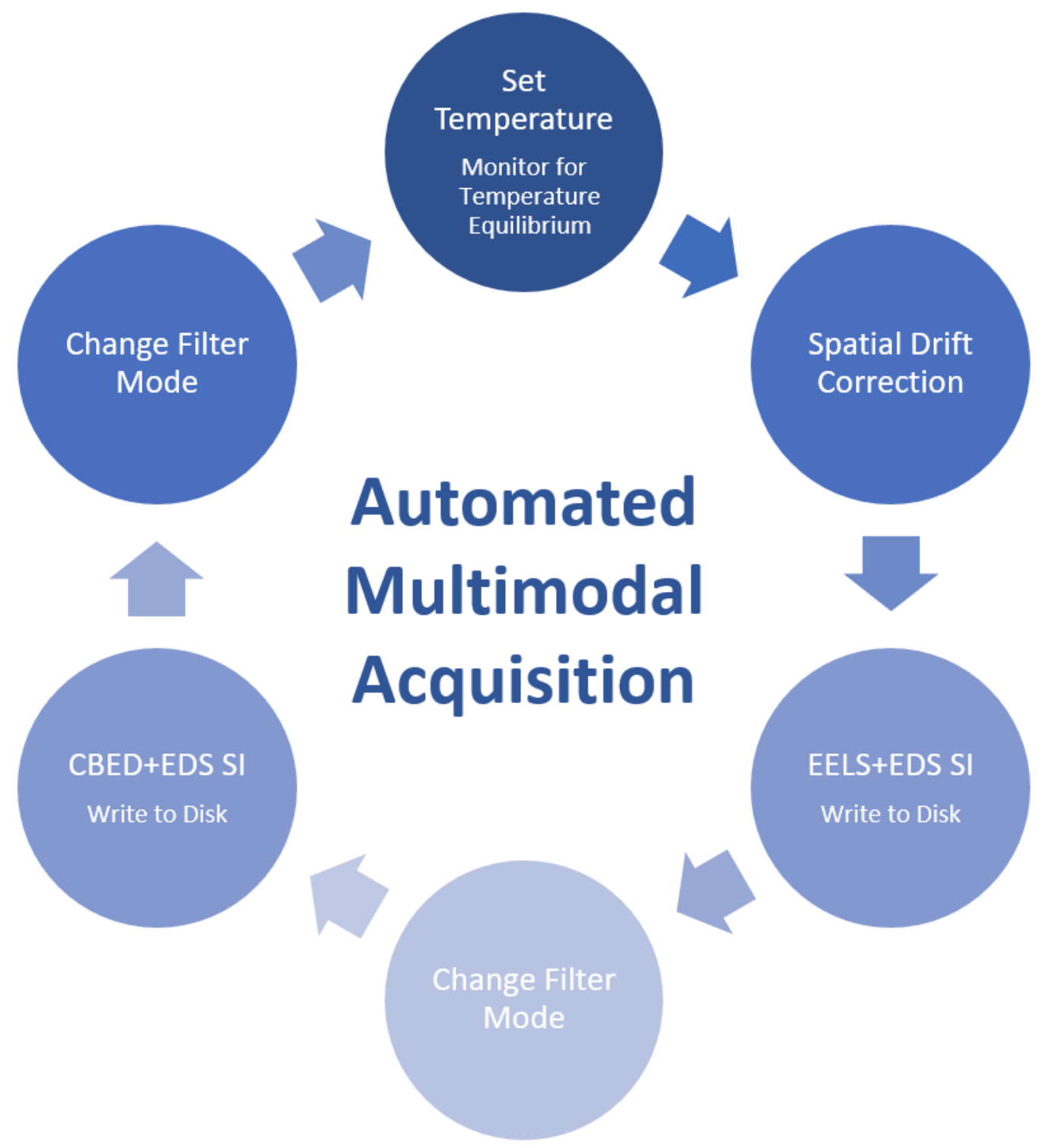

Figure 1. Figure 1 - Flow diagram showing data acquisition and holder control steps in the multimodal multiple pass spectrum imaging method. 


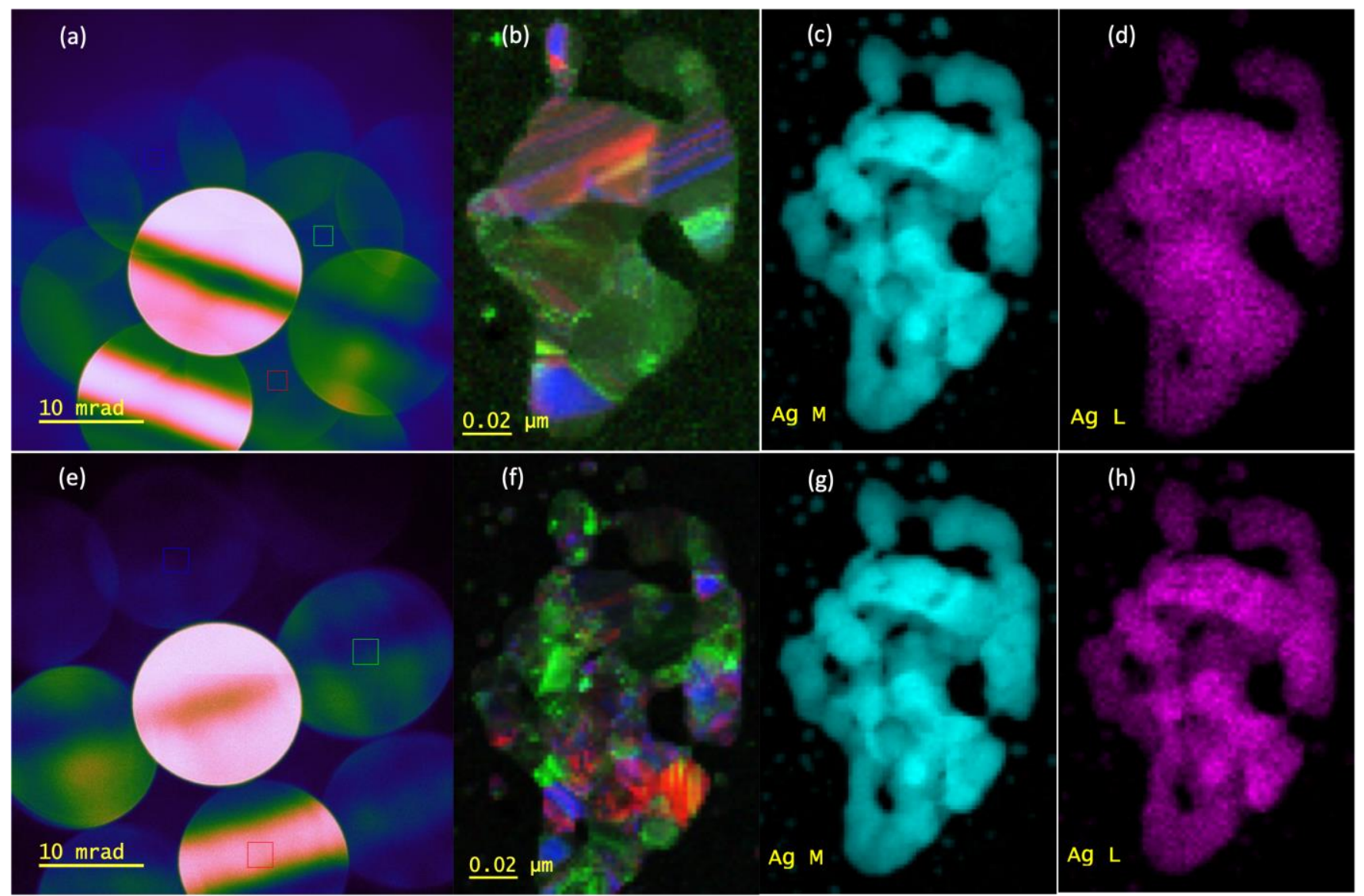

Figure 2. Figure 2 - Multimodal SI data acquired from Silver (II) oxide powder at $600^{\circ} \mathrm{C}$ : (a) energyfiltered CBED pattern showing virtual aperture positions for (b) orientation map. (c) EELS Ag M-edge map, and (d) EDS Ag L-peak map. Equivalent data for $25^{\circ} \mathrm{C}$ is also shown (e-h).

References

[1] L. Spillane, et al., Microsc. Microanal. 26 (Suppl 2), (2020) 